\title{
Tipo de Reforçador como Variável Moduladora dos Efeitos da História em Humanos
}

\author{
Carlos Eduardo Costa \\ Universidade Estadual de Londrina \\ Roberto Alves Banaco \\ Pontifícia Universidade Católica de São Paulo \\ Denise M. Longarezi \\ Everton V. Martins \\ Evelise M. Maciel \\ Camila H. Sudo \\ Universidade Estadual de Londrina
}

\begin{abstract}
RESUMO - Para investigar como o tipo de reforçador afeta o comportamento em FI após diferentes histórias de reforço, universitários foram expostos a FR ou DRL, que liberavam pontos por pressionar um botão. Alguns participantes trocavam pontos por fotocópias (Condição 1), por dinheiro (Condição 2) ou apenas recebiam os pontos (Condição 3). Subseqüentemente, todos foram expostos a FI. O FR produziu taxas de respostas altas e constantes independentemente do tipo de reforçador utilizado. O FI produziu taxas altas para os participantes das Condições 1 e 2 e baixas para os participantes da Condição 3. O DRL produziu baixas taxas que aumentaram durante 0 FI subsequiente. Os resultados sugerem que tanto contingências históricas quanto presentes controlaram o comportamento dos participantes e que o tipo de reforçador pode favorecer o responder em taxa alta e constante sob FI após exposição a uma contingência de FR.
\end{abstract}

Palavras-chave: efeitos da história; história de reforço; história comportamental; esquemas de reforçamento; tipo de reforçador; comportamento humano.

\section{The Type of Reinforcer as a Modulating Variable of History in Humans Effects}

\begin{abstract}
To investigate how the type of reinforcer affects the behavior in FI after different reinforcement histories, university students were exposed to FR or to DRL, that liberated points for pressing a button. Some participants changed points for photocopies (Condition 1), for money (Condition 2), or they just received the points (Condition 3). Subsequently, all students were exposed to FI. FR produced high and constant response rates independently of the type of reinforcer. FI produced high rates for the participants of the Conditions 1 and 2, and low for the participants of the Condition 3. DRL provoked low rates that increased during subsequent FI. The results suggest that so much historical contingencies as present contingencies controlled the participants' behavior and that the type of reinforcer can favor responding in high and constant rate under FI after a FR contingency.
\end{abstract}

Key words: history effects; history of reinforcement; behavioral history; schedules of reinforcement; type of reinforcers; human behavior.

O comportamento de um organismo é função de variáveis ambientais presentes no momento em que ele responde e de sua história de condicionamento (Baum, 1999; Morse \& Kelleher, 1970; Skinner, 1953; 1966). Estudos experimentais do efeito que a história de reforço exerce sobre o comportamento atual evoluíram - como área de pesquisa - de modo bem menos sistemático do que os estudos do efeito que os programas de reforço presentes em uma situação exercem sobre o comportamento (Tatham \& Wanchisen, 1998).

Alguns autores (Wanchisen, Tatham \& Mooney, 1989; Wanchisen, 1990; Wanchisen \& Tatham, 1991; Weiner, 1983) defendem a idéia de que os estudos acerca do efeito de experiências passadas sobre o comportamento presente podem lançar luz sobre uma questão bastante controvertida na literatura experimental: a diferença encontrada em pesquisas experimentais sobre o padrão do responder de humanos e não-humanos em programas de reforço em geral e, especi-

1 Endereço: Rua da lapa, 498, Apto 101, Jardim Higienópolis, Londrina, PR, Brasil 86015-050 E-mail: caecosta@uel.br. ficamente, quando expostos a um programa de reforço em intervalo fixo (FI).

Em um programa de reforço em FI, a primeira resposta emitida após um período designado de tempo é seguida por um evento reforçador (Catania, 1998; Ferster \& Skinner, 1957; Lattal, 1991). Dois padrões de responder sob uma contingência de FI têm sido mais comumente relatados na literatura experimental com organismos não-humanos: o scallop - que consiste de uma pausa logo após o reforço seguido por um responder positivamente acelerado dentro do intervalo até a liberação do próximo reforço (Ferster \& Skinner, 1957) - e o break-andrun, que consiste de uma pausa, geralmente após o reforço, e uma transição abrupta na taxa de resposta de uma frequiência próxima de zero para uma freqüência constante que é mantida até o final do intervalo (Cumming \& Schoenfeld, 1958). Alguns estudos experimentais com humanos (Weiner, 1965, 1969) indicaram que sob um programa de FI, o padrão de responder entre reforços sucessivos é, comumente, de taxa de resposta alta e constante ou de taxa baixa (geralmente com uma pausa, que dura quase todo o intervalo, e poucas respostas no final). 
De modo geral, considerações a respeito dessa diferença no padrão de responder entre humanos e não-humanos e a respeito da variabilidade nos resultados entre participantes encontrada nas pesquisas com humanos apontam para pelo menos três direções (não mutuamente excludentes): a) o papel do comportamento verbal - mais especificamente, alguns autores (Catania, 1998; Lowe, 1979) defendem que as instruções e auto-instruções podem tornar o comportamento humano insensível às contingências de reforço presentes; b) diferenças nos procedimentos experimentais empregados nas pesquisas com humanos e nãohumanos e mesmo entre as diferentes pesquisas com humanos (Perone, Galizio \& Baron, 1988) ${ }^{2}$, como por exemplo, modo de aquisição da resposta operante (modelagem vs. instrução - Matthews, Shimoff, Catania \& Sagvolden, 1977); a exigência ou não de uma resposta de consumação (Buskist, Miller \& Bennett, 1980; Matthews \& cols., 1977; Raia, Shillingford, Miller \& Baier, 2000); o reforçador empregado, pode-se citar como alguns exemplos as possíveis diferenças no emprego de reforçadores primários (como feito na maioria das pesquisas com não-humanos) vs. Reforçadores secundários (Stoddard, Sidman \& Brady, 1988; Matthews \& cols., 1977; Weiner, 1964; 1969), além das diferenças quanto à duração da exposição às contingências de reforço, grau de controle da história extra-experimental, etc.; e c) o papel da história de reforço.

Por exemplo, Weiner (1964) investigou os efeitos de duas histórias de reforço sobre o comportamento humano de pressionar um botão sobre um segundo programa de reforço subseqüente. Participaram do experimento seis indivíduos, pagos por hora, que foram distribuídos por dois grupos. Os participantes de um grupo foram submetidos a 10 sessões de uma hora de FR 40 e os participantes de outro grupo realizaram 10 sessões de uma hora de DRL 20s. Em seguida, todos os participantes foram submetidos a 10 sessões de uma hora e mais 10 sessões de 30 minutos, todas em FI 10s. Luzes de diferentes cores foram correlacionadas a cada um dos programas de reforço utilizados. Sob o programa de FI, os participantes que haviam sido submetidos a FR 40 emitiram taxas relativamente mais altas de respostas do que os participantes que foram submetidos a DRL 20s, o que sugere que a história de condicionamento pode ser considerada uma possível determinante do responder humano em FI. Apesar de os participantes com história de FR terem pressionado o botão em taxas sempre superiores aos participantes com história de DRL, as taxas de respostas daqueles com história de FR tenderam a diminuir nas últimas sessões de FI e as taxas de respostas daqueles com história de DRL tenderam a aumentar. Resultados semelhantes foram relatados por Urbain, Poling, Millam e Thompson (1978), LeFrancois e Metzger (1993) e Baron e Leinenweber (1995) com ratos e Freeman e Lattal (1992) com pombos.

Uma característica importante de vários dos estudos de Weiner $(1964,1965,1969)$ é o fato de os participantes ganharem dinheiro por hora de sessão e não pelo desempenho

2 Como salientaram Perone e cols. (1988) os resultados aparentemente mais regulares que emergem de estudos com organismos não-humanos provêm de um conjunto de procedimentos-padrão de laboratório que não encontra, até o momento, contrapartida nos estudos com humanos. Ademais, segundo os mesmos autores, variabilidade inexplicável é também comum - e muitas vezes negligenciada - em estudos com organismos não-humanos. Tal negligência pode pintar um quadro exaltado e um tanto irreal do que é conhecido sobre o condicionamento operante. exibido durante as sessões. A esse respeito, Weiner (1970) apresenta resultados que sugerem que a persistência de um responder em taxa alta e constante sob um FI 10s-custo, após uma história de responder em FR 40, é obtido com participantes cujos pontos eram trocáveis por dinheiro (U\$ 0,01 por ponto) e participantes cujos pontos não eram trocáveis por nada. Sob a contingência de FI 10s-custo os participantes recebiam 100 pontos para a primeira resposta emitida após um intervalo de 10s e perdiam um ponto para cada resposta emitida durante o intervalo entre reforços. Outros resultados indicaram que mesmo quando o tipo de reforço foi alterado entre as sessões de FI 10s-custo, a persistência do responder em taxa alta e constante se manteve após uma história de FR, mas não quando uma história de DRL foi adicionada. Os participantes foram expostos primeiramente a um programa de FR 40, no qual ganharam U\$ 2,00 por sessão e o reforço durante a sessão foram apenas os pontos. Depois, os participantes foram submetidos a três condições de FI 10s-custo em seqüência. Na Condição 1, os participantes ganharam U\$2,00 por sessão e os reforços foram apenas os pontos; na Condição 2, os participantes ganharam U\$ 2,00 por sessão e os reforços foram pontos trocáveis por U\$ 0,01 por ponto; na Condição 3, os participantes não ganharam nada pela sessão e os reforços foram pontos trocáveis por U\$ 0,01. Após essas três condições de FI-custo, os participantes foram expostos a um programa de reforço em DRL 20s-custo, no qual não ganharam por sessão e os reforços foram pontos trocáveis por U\$ 0,01 , e então foram submetidos novamente à Condição 3 do FI 10s-custo. Os resultados indicaram que o padrão de responder em taxa alta e constante se manteve nas três condições do FI 10 s-custo, mas após a exposição ao programa de DRL 20s-custo, os participantes passaram a exibir um padrão de responder em taxa baixa como aquelas exibidas durante o DRL-custo (Weiner, 1970). O autor argumentou que esses dados intraparticipantes sugerem que a contingência de reforço pode ser mais efetiva em produzir mudanças comportamentais do que o tipo de reforçador utilizado.

Que as contingências de reforço são determinantes poderosos do comportamento (cf. Morse \& Kelleher, 1970; Zeiler, 1977) parece não haver dúvidas. Todavia, em todas as condições do estudo de Weiner (1970), os participantes ganharam algum dinheiro (por sessão, pelos pontos obtidos ou ambos). Uma condição na qual o participante recebesse apenas os pontos (que não fossem trocáveis por nada) talvez produzisse efeitos que, no estudo de Weiner, podem ter sido encobertos pelos ganhos obtidos por sessão ou pelos pontos. Em segundo lugar, Stoddard, Sidman e Brady (1988) relataram resultados que indicaram que o desempenho de humanos foi sensível às mudanças nas contingências de reforço durante o experimento. Os autores sugeriram que o tipo de reforçador empregado, fichas trocáveis por mercadorias, que contribuíam para um "aumento substancial no salário líquido" (p. 34) dos participantes foi uma variável importante que, provavelmente, "aumentou a sensibilidade [do comportamento] dos sujeitos às contingências" (p. 42). Em terceiro lugar, dados do próprio Weiner (1972) sugerem que a forma como o participante recebe dinheiro durante o experimento pode alterar substancialmente seu desempenho sob um programa de reforço em FR. Participantes cujos pontos eram trocados por dinheiro emitiram taxa de resposta mais alta do que quando os pontos não eram trocados por nada, isto é, os participantes ganhavam apenas 
por sessão ou não ganhavam nada. Por fim, como afirmaram Critchfield, Schlund e Ecott (2000), encontrar reforçadores efetivos tem sido um dos desafios em se conduzir análises experimentais do comportamento com humanos.

O presente experimento teve por objetivo verificar se o tipo de reforçador empregado poderia exercer algum efeito sobre o desempenho de humanos sob uma contingência de FI, após uma história de responder sob FR ou DRL.

\section{Método}

\section{Participantes}

Participaram do experimento 20 universitários (13 homens e sete mulheres), da Universidade Estadual de Londrina. Nenhum dos participantes era do curso de Psicologia. Alguns dos participantes pertenciam à mesma turma de um mesmo curso. Apesar de ser solicitado aos participantes que não conversassem com outras pessoas sobre as sessões experimentais, nenhum tipo de controle foi realizado a esse respeito.

\section{Equipamentos e Instrumentos}

Microcomputadores: Foram utilizados três microcomputadores do tipo PC com monitor de 14 polegadas, mouse e teclado padrão e Windows ${ }^{\circledR} 95$ e 98 se.

Instrumentos: Foram utilizados os softwares ProgRef v2 (Costa \& Banaco, 2002a) e ProgRef v3 (Costa \& Banaco, 2002b, 2003). As duas versões do software foram programadas em linguagem Visual Basic ${ }^{\circledR} 6.0$ e eram executáveis em ambiente Windows®. O layout da tela, a lógica da programação das contingências de reforço e o registro dos dados principais - inclusive o gráfico de registro cumulativo - eram idênticos para as duas versões do software. A diferença mais importante consistiu no fato de que a terceira versão permitiu o registro dos IRTs (intervalos entre respostas) de todas as respostas dos participantes. Testes realizados antes do início do procedimento experimental revelaram que não havia qualquer diferença detectável no desempenho do software, em suas duas versões entre os três microcomputadores e versão do Windows ${ }^{\circledR}$ (95 e 98se) utilizada.

Os participantes deviam clicar com o cursor do mouse sobre um retângulo (botão de resposta) que aparecia no monitor (resposta descrita apenas como "pressionar o botão" daqui para frente). Os pontos apareciam em uma janela (visor de pontuação) acima do botão de resposta e eram apresentados de acordo com o programa de reforço selecionado pelo experimentador. A Figura 1 exibe um layout da tela do computador com a qual os participantes interagiram.

Quando o critério para o cumprimento da contingência de reforço em vigor era cumprida, aparecia no canto superior direito do monitor um ícone identificado comumente como um "smile". O participante devia, então, clicar com a seta do mouse sobre o botão que se localizava no canto superior direito da tela (o botão da resposta de consumação), acima desse ícone. Ao fazer isso, o smile desaparecia e o um ponto era creditado no contador. Enquanto um smile estivesse presente no monitor, não aparecia outro até que o participante pressionasse o botão da resposta de consumação. Depois que o smile desaparecia-e o ponto era creditado -, o participante, caso voltasse a pressio-

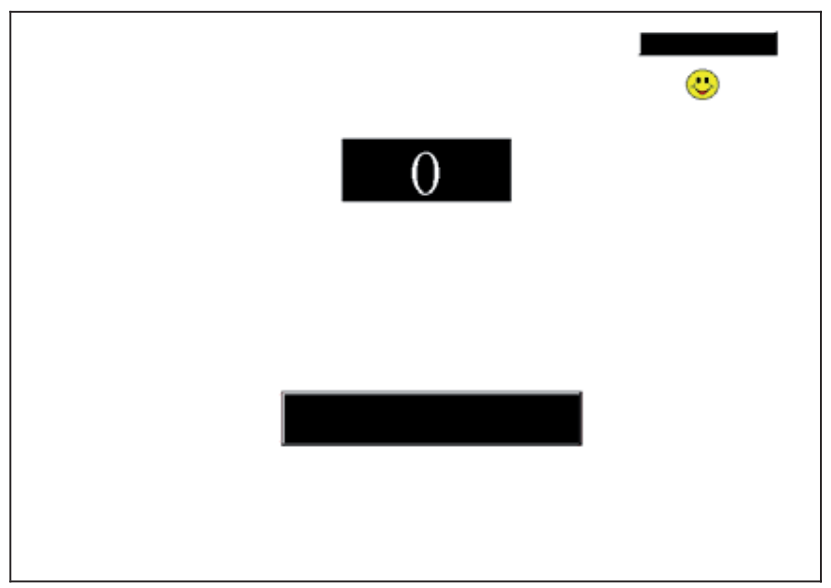

Figura 1. Layout da tela do computador durante as sessões experimentais.

nar o botão de resposta, poderia ganhar mais pontos. Caso o participante continuasse a pressionar o botão de respostas após o aparecimento do smile, as respostas eram registradas. Portanto, se um programa de reforço em FR, por exemplo, estivesse em vigor, as respostas que eventualmente ocorressem após o aparecimento do smile eram contadas para o cumprimento da contingência e a liberação do ponto seguinte. Os cronômetros que controlavam a execução do software continuavam em operação durante todo o experimento, ou seja, o tempo consumido pelo participante para pressionar o botão de resposta de consumação era computado como parte do intervalo entre reforços. O intervalo de um FI, por exemplo, era iniciado a partir do aparecimento do smile (liberação do reforço), e não a partir da emissão da resposta de consumação.

A resposta de consumação tinha a função de manter o participante olhando em direção ao monitor. Sem essa resposta, o participante poderia, por exemplo, manter-se pressionando o botão durante toda a sessão e olhar para os pontos obtidos (i.e., fazer contato com a conseqüência programada) apenas ao final da sessão. Além disso, pesquisas (e.g. Matthews \& cols., 1977; Raia \& cols., 2000) apontaram para a importância da resposta de consumação no estabelecimento de um responder aparentemente sensível ao parâmetro temporal da contingência de reforço (em FI ou VI) em humanos.

\section{Local}

As sessões experimentais foram realizadas em duas salas do Departamento de Psicologia Geral e Análise do Comportamento do Centro de Ciências Biológicas da Universidade Estadual de Londrina. Apenas um participante era colocado em cada sala por vez.

\section{Reforçadores}

Foram utilizados como reforçadores: (1) pontos trocáveis por dinheiro, (2) pontos trocáveis por fotocópias ou (3) pontos apenas.

\section{Procedimento}

Antes do início da primeira sessão, o participante lia e assinava o Termo de Consentimento Livre e Esclarecido 
(TCLE) $)^{3}$. No TCLE, os participantes eram informados se os pontos obtidos na sessão seriam trocados por dinheiro ou fotocópias. Caso o participante fosse da condição cujos pontos não eram trocados por nada, nenhuma informação era dada sobre a troca dos pontos. Ao adentrar pela primeira vez a sala onde era realizado o experimento, o participante lia as seguintes instruções em uma folha:

\section{Obrigado por sua colaboração!}

Sua tarefa será clicar com o botão esquerdo do mouse sobre um retângulo que aparece na parte inferior do monitor para obter pontos.

Abaixo você pode observar o layout da tela.

[Uma figura idêntica à da Figura 1 era apresentada]

Você deve tentar ganhar o maior número de pontos que você conseguir. Você ganhará pontos clicando sobre o botão de uma maneira específica.

Os pontos aparecerão na janela que se localiza na parte superior da tela na posição central. Entretanto, quando você ganhar algum ponto aparecerá no canto superior direito do monitor um smile. Você deverá, então, clicar com a seta do mouse sobre o botão que se localiza no canto superior direito da tela. Ao fazer isso o smile desaparecerá e o ponto será creditado no contador. Quando um smile aparecer no monitor não aparecerá outro até que você clique no botão menor no canto superior direito. Depois que o smile desaparece - e o ponto é creditado - você pode voltar a clicar sobre o botão maior na parte inferior do monitor para ganhar mais pontos.

Boa sorte!

Em seguida, era solicitado que o participante deixasse todo seu material, inclusive relógio, sobre uma mesa distante (a pelo menos dois metros) do computador no qual ele trabalhava. Também era solicitado que os participantes

Tabela 1. Distribuição dos participantes pelos grupos e condições do experimento e seqüência das etapas.

\begin{tabular}{|c|c|c|c|c|}
\hline Grupo & Condição & $\begin{array}{l}\text { Etapa } 1 * \\
(1 \text { ou } 2)^{* *}\end{array}$ & $\begin{array}{c}\text { Etapa } 2 \\
\text { (3) }\end{array}$ & $\begin{array}{c}\text { Etapa } 3 \\
\text { (3) }\end{array}$ \\
\hline \multirow{3}{*}{$\begin{array}{c}\text { FR } \\
(n=11)\end{array}$} & $\begin{array}{l}\text { 1. Fotocópias } \\
\quad(\mathrm{n}=3)\end{array}$ & \multirow{3}{*}{$\begin{array}{c}\text { Treino em } \\
\text { FR }\end{array}$} & \multirow{3}{*}{ FR 40} & \multirow{3}{*}{ FI 10s } \\
\hline & $\begin{array}{l}\text { 2. Dinheiro } \\
(\mathrm{n}=3)\end{array}$ & & & \\
\hline & $\begin{array}{l}\text { 3. Pontos } \\
(\mathrm{n}=5)\end{array}$ & & & \\
\hline \multirow{3}{*}{$\begin{array}{c}\text { DRL } \\
(\mathrm{n}=9)\end{array}$} & $\begin{array}{l}\text { 1. Fotocópias } \\
(\mathrm{n}=3)\end{array}$ & \multirow{3}{*}{$\begin{array}{c}\text { Treino em } \\
\text { DRL }\end{array}$} & \multirow{3}{*}{ DRL 20s } & \multirow{3}{*}{ FI $10 \mathrm{~s}$} \\
\hline & $\begin{array}{l}\text { 2. Dinheiro } \\
(\mathrm{n}=3)\end{array}$ & & & \\
\hline & $\begin{array}{l}\text { 3. Pontos } \\
(\mathrm{n}=3)\end{array}$ & & & \\
\hline
\end{tabular}

* Durante a Etapa 1 a consequiência programada foi apenas os pontos para todos os participantes. Somente a partir do início da Etapa 2 as consequiências programadas eram aquelas descritas na segunda coluna da esquerda para a direita.

** Os números entre parênteses, abaixo das Etapas, indicam o número de sessões que foram realizadas.

3 O experimento aqui descrito foi aprovado pelo Comitê de Ética em Pesquisa com Seres Humanos da Universidade Estadual de Londrina. desligassem seus celulares, caso estivessem portando algum. A Tabela 1 sumaria as etapas do experimento.

Cada participante foi designado para uma condição (Fotocópias, Dinheiro ou Pontos) - referente ao tipo de consequiência programada para o desempenho nas sessões - de um dos grupos (Grupo FR ou Grupo DRL) - referente à história de reforço.

Condição 1. Fotocópia: os participantes recebiam, ao final de cada sessão, um "crédito" de uma fotocópia no Centro Acadêmico do curso de Psicologia para cada ponto obtido nas sessões. À época do experimento, cada fotocópia custava R\$ 0,05.

Condição 2. Dinheiro: os participantes recebiam, ao final de cada sessão, $\mathrm{R} \$ 0,05$ para cada ponto obtido nas sessões.

Condição 3. Pontos: os participantes não ganhavam nada pelos pontos obtidos nas sessões.

Diferentemente de alguns dos estudos realizados por Weiner (e.g. Weiner, 1964, 1969, 1970), em que os participantes ganhavam dinheiro por sessão experimental ou por hora de participação no experimento, em nenhum dos casos do presente estudo, os participantes ganharam dinheiro (ou qualquer outra coisa) por participação nas sessões experimentais. Os ganhos eram relativos exclusivamente aos pontos obtidos ou, no caso dos participantes da Condição 3, nenhum ganho estava programado.

Etapa 1. Imediatamente após a leitura da instrução os participantes foram submetidos a uma sessão de treinamento do comportamento de pressionar o botão do mouse sob um programa de reforço em FR ou DRL. Essas sessões - denominadas de treino - consistiram de várias exposições curtas à contingência de reforço. Por exemplo, para os participantes expostos ao FR, o valor da razão era aumentado a cada três minutos na seguinte sequiência: FR 5, 10, 15, 20 e 40. No final de cada período de três minutos, uma mensagem aparecia na tela do computador, informando ao participante quantos pontos ele havia obtido e que ele deveria chamar o experimentador. O experimentador entrava na sala e programava as contingências para o próximo período de três minutos de exposição ao programa de reforço. Os participantes do Grupo DRL eram submetidos a uma ou duas sessões de treinamento em DRL. O valor do intervalo era aumentado, a cada três minutos, na seguinte seqüência: $2,3,5$, 10,15 e 20 segundos. Ao final de cada período de três minutos, uma mensagem aparecia na tela do computador, informando ao participante quantos pontos ele havia obtido e que ele deveria chamar o experimentador, que entrava na sala experimental e programava um novo período de três minutos.

Durante essa etapa, todos os participantes tinham como consequiência programada pelo seu desempenho apenas os pontos. Somente a partir da Etapa 2 as consequiências programas foram aquelas descritas anteriormente: pontos trocáveis por fotocópias (Condição 1), pontos trocáveis por dinheiro (Condição 2) ou pontos apenas (Condição 3).

Etapa 2. Os participantes eram expostos a três sessões de 15 minutos cada, com o programa de reforço específico de cada grupo. Os participantes do Grupo FR foram expostos a um programa de reforço em FR 40, no qual a 40ª resposta emitida desde o reforço prévio (ou do início da sessão) era seguida pelo aparecimento do smile. Os participantes do Grupo DRL foram expostos a um programa de reforço em DRL 20s, no qual a primeira resposta emitida após um intervalo de $20 \mathrm{~s}$ desde a resposta prévia (ou do início da sessão) era seguida pelo aparecimento do smile. Respostas que ocorriam antes 
que o intervalo de 20s tivesse passado zeravam o cronômetro e o intervalo começava a ser contado novamente.

Etapa 3. Os participantes de ambos os grupos foram submetidos a um programa de reforço em FI 10s, no qual a primeira resposta que ocorresse após um intervalo de 10s desde o reforço prévio (ou do início da sessão) era seguida pelo aparecimento do smile. Respostas que ocorressem antes de completado os 10s não tinham nenhuma consequiência programada. Foram realizadas três sessões de 15 minutos cada nessa etapa.

Assim como nos estudos de Weiner (1964, 1965, 1969, 1970), nos quais luzes de cores distintas estavam acesas para cada contingência envolvida no experimento, a cor do botão de respostas mudou entre as Etapas 2 e 3 do presente experimento. Os participantes da Condição 1 - Fotocópia e da Condição 2 - Dinheiro utilizaram a versão 2 do software (que não fazia o registro dos IRTs) e os participantes da Condição 3 - Pontos utilizaram a versão 3 (que fazia o registro dos IRTs). Isso ocorreu porque a decisão de incluir mais um tipo de reforço no experimento foi tomada após o início da coleta de dados dos participantes das outras duas condições e, nessa ocasião, a nova versão do software estava pronta. As sessões experimentais eram realizadas de segunda à sexta-feira (exceto feriados) - pelo menos duas vezes por semana - no período das 08:00 às 18:00h, de acordo com a disponibilidade dos participantes.

\section{Resultados}

\section{Desempenho sob FI 10s dos participantes submetidos a uma história experimental de FR}

A Figura 2 exibe a freqüência acumulada de pressão ao botão dos participantes S1 e S3 da Condição 1 - Fotocópias, com história de responder em FR, na terceira sessão de exposição ao programa de reforço em FR 40 e nas três sessões de reforço em FI 10s. O padrão de respostas do participante S1 é representativo do desempenho do participante S2 (da Condição 1 - Fotocópias) e dos participantes S11, S12 e S13 (da Condição 2 - Dinheiro). Apesar de o participante S3 ter emitido taxas de respostas menores que os demais participantes é possível afirmar que, de maneira geral, os participantes da Condição 1 - Fotocópia e da Condição 2 - Dinheiro exibiram um padrão de desempenho em taxa alta e constante durante a exposição ao FR 40 e mantiveram esse padrão de desempenho quando a contingência mudou para FI 10s.

Nota-se também um aumento na freqüência de resposta entre as sessões do participante S3 (i.e. 1.538 respostas na terceira sessão do FR e 2.484 respostas na terceira sessão de exposição ao FI 10s). O aumento do número de respostas foi acompanhado por um aumento no número de pontos obtidos quando a contingência mudou de FR para FI, mas não durante a exposição ao FI.

A Figura 3 exibe a freqüência acumulada de pressão ao botão dos participantes da Condição 3 - Pontos na terceira sessão de exposição ao programa de reforço em FR 40 e nas três sessões de reforço em FI 10s.

Nota-se, na Figura 3, que o desempenho de todos os participantes da Condição 3 - Pontos foi de taxa alta na última sessão de exposição ao FR 40. Com a mudança na contingência de FR para FI, houve uma redução na taxa de respostas de quatro dos cinco participantes. Apenas o participante S21 manteve o mesmo padrão comportamental de alta taxa durante as três sessões de FI 10s. Para os participantes S22 e S24, a mudança de um padrão de taxa alta para taxa mais baixa foi precedida por oscilações entre taxa de resposta alta e baixa, como pode ser observado

\section{Condição 1 - Fotocópias}

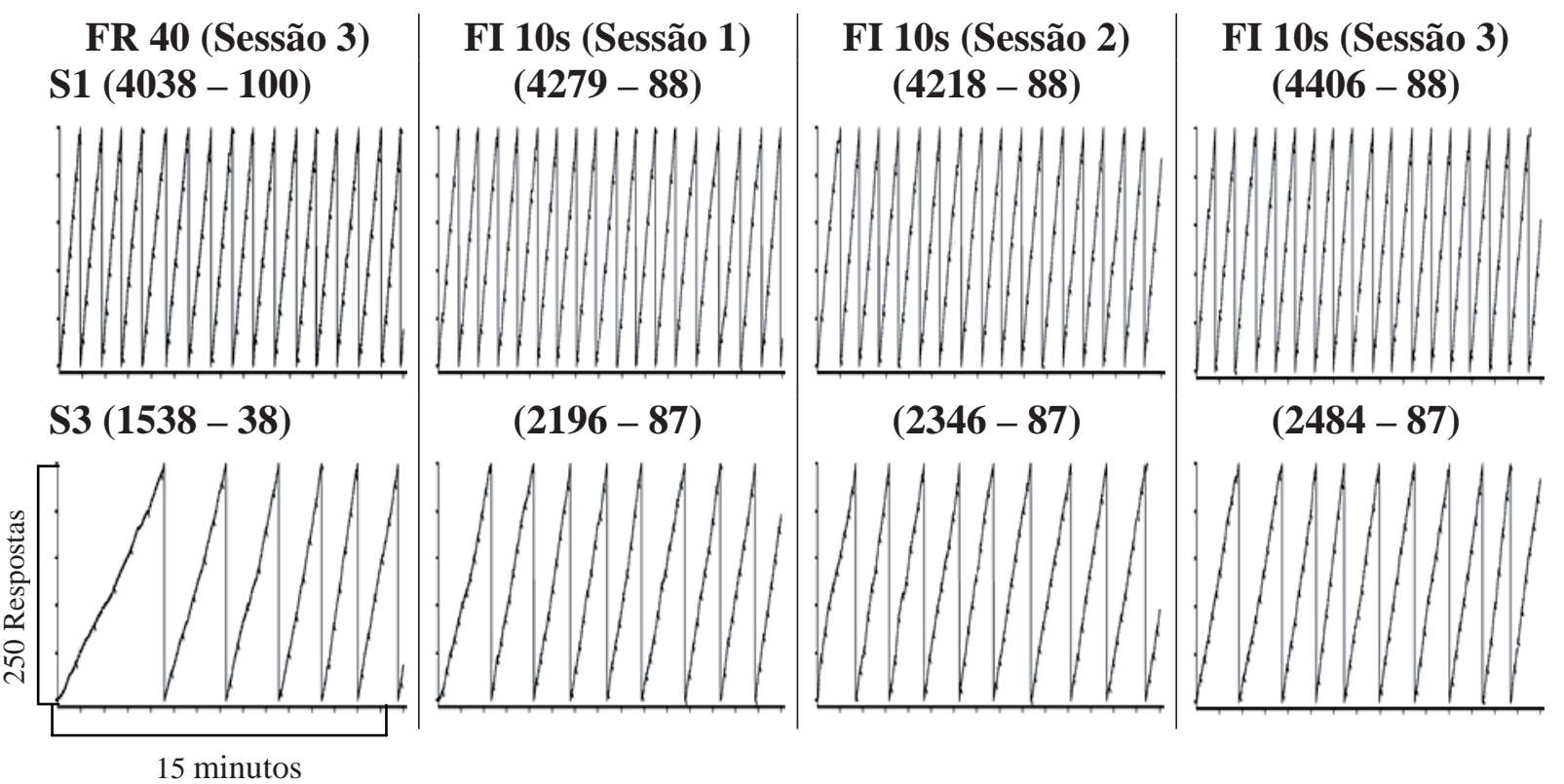

Figura 2. Freqüência acumulada de pressão ao botão dos participantes S1 e S3 (Condição 1-Fotocópia) na terceira sessão de exposição a um programa de reforço em FR 40 (primeira coluna da esquerda) e nas três sessões de exposição a um programa de reforço em FI 10s. Os registros cumulativos voltam a zero após 250 respostas e as marcas diagonais nos registros indicam a ocorrência de reforço. Todas as sessões tiveram duração de 15 minutos. Os números entre parênteses, acima de cada registro cumulativo, referem-se ao total de respostas e ao número de pontos obtidos naquela sessão. 


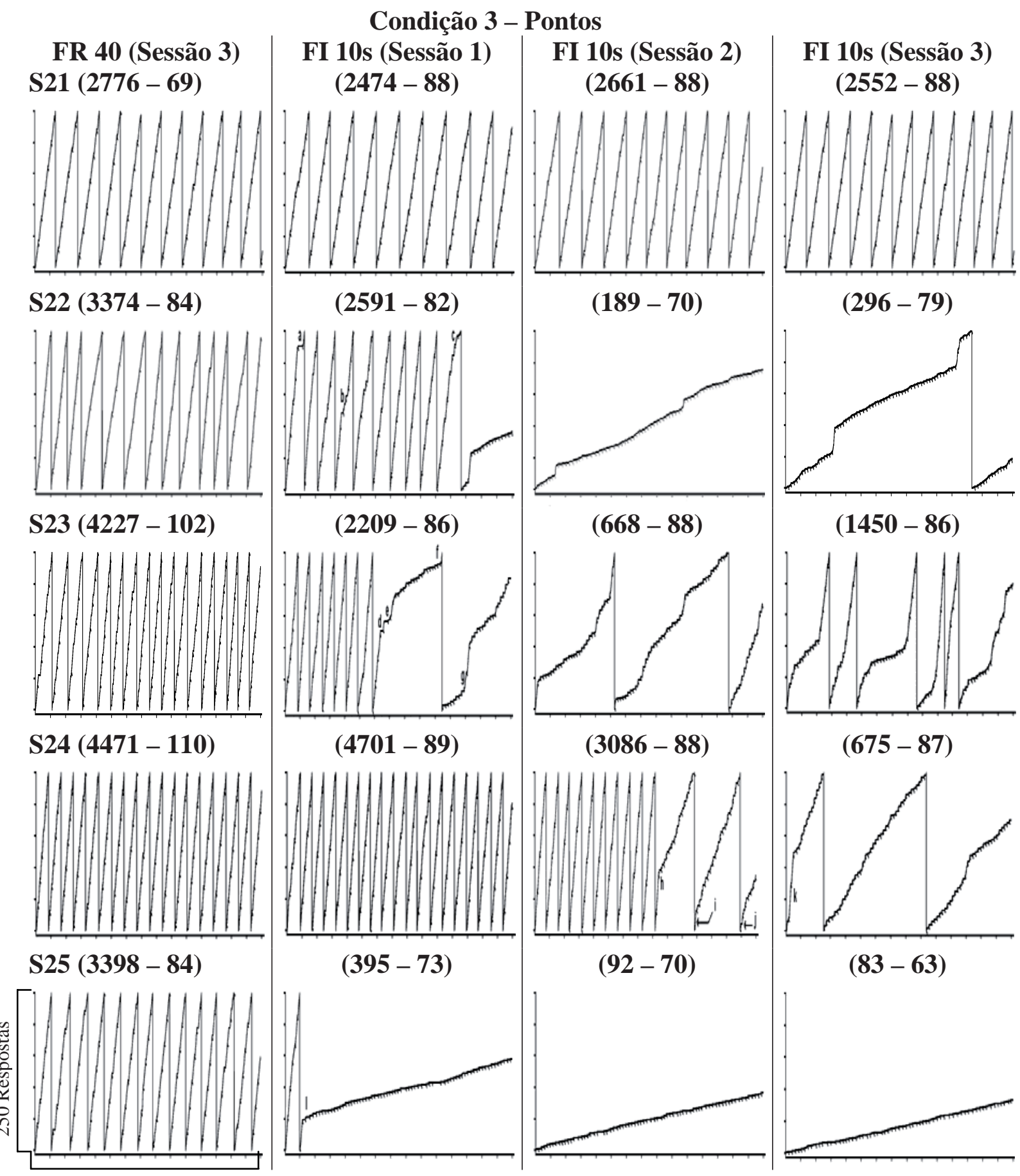

Figura 3. Freqüência acumulada de pressão ao botão dos participantes S21, S22, S23, S24 e S25 (Condição 3-Pontos) na terceira sessão de exposição a um programa de reforço em FR 40 (primeira coluna da esquerda) e nas três sessões de exposição a um programa de reforço em FI 10s (segunda, terceira e quarta colunas). Os registros cumulativos voltam a zero após 250 respostas e as marcas diagonais nos registros indicam a ocorrência de reforço. Todas as sessões tiveram duração de 15 minutos. Os números entre parênteses, acima de cada registro cumulativo, referem-se ao total de respostas e ao número de pontos obtidos naquela sessão.

nas letras $a, b$, e $c$ (S22) e nas marcas $h, i$ e $j(\mathrm{~S} 24)$, na Figura 3. O participante $\mathrm{S} 24$ apresentou um padrão de responder em taxa alta no início da terceira sessão do FI, voltando em seguida ao padrão de taxa mais baixa após o primeiro minuto de sessão (marca $k$ na Figura 3). O participante S23 começou a apresentar oscilações entre taxa alta e baixa também na primeira sessão de exposição ao FI, como pode ser observado nas marcas $d, e$, $f$ e $g$ da Figura 3 e manteve esse padrão até a terceira sessão de exposição ao FI 10s. O participante S25 alterou o padrão comportamental de taxa alta para taxa mais baixa de modo aparentemente abrupto logo na primeira sessão de exposição ao FI, como pode ser observado na marca $l$, na Figura 3.

Um padrão observado nos registros cumulativos da terceira sessão de exposição ao FI 10s dos participantes da 
Condição 3 - Pontos do Grupo FR foi que, após o reforço (smile), umas poucas respostas eram ainda emitidas e, então, uma pausa ocorria seguida pelo retorno de um responder constante (maiores detalhes na Figura 4). Esse padrão poderia estar relacionado ao tipo de programação da resposta de consumação. Pouco antes de cada reforço alguns participantes (S21, S23 e S24) estavam emitindo taxa de respostas alta e constante; quando o reforço era liberado esses participantes ainda emitiam algumas respostas (de duas a quatro respostas, aproximadamente) antes de pressionarem o botão da resposta de consumação. A pequena pausa que ocorre em seguida é de-

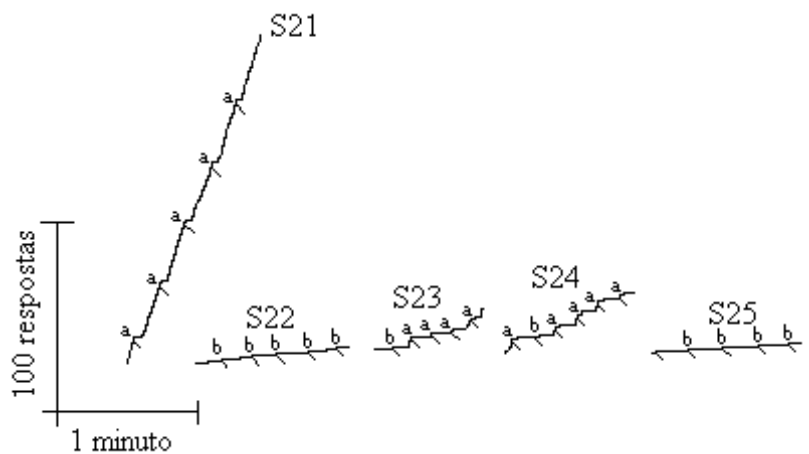

Figura 4. Freqüência acumulada de respostas de pressão ao botão da

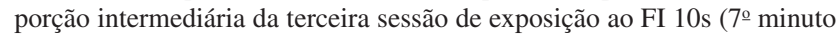
da sessão) dos participantes da Condição 3-Pontos do Grupo FR. As marcas diagonais nos registros cumulativos indicam a ocorrência de reforço. As marcas a indicam exemplos de taxa alta de resposta ocorrendo no final de um intervalo e "invadindo" o início do intervalo subseqüente. As marcas b indicam exemplos da ausência desse padrão quando a taxa de resposta no final de um intervalo é baixa.

vida ao tempo consumido pelos participantes para deslocarem o cursor do mouse do botão de resposta até o botão da resposta de consumação e voltar com o cursor até o botão de respostas.

Devido ao tipo de programação da resposta de consumação, a distribuição das respostas entre reforços consecutivos em um dado intervalo foi "invadida" pela taxa de respostas final do intervalo precedente. Para clarificar melhor esse fato, a Figura 4 exibe os registros cumulativos de pressão ao botão da porção intermediária (o sétimo minuto) da terceira sessão de exposição ao FI 10s dos participantes da Condição 3 - Pontos do Grupo FR.

É possível observar com maiores detalhes, na Figura 4, que, quando o desempenho dos participantes no final de algum intervalo é de taxa alta, algumas respostas são emitidas no início do intervalo posterior (como pode ser visualizado nas marcas $a$ na Figura 4). Todavia, quando o padrão de responder no final de um intervalo é de taxa baixa (geralmente em intervalos com aproximadamente duas respostas), o intervalo subseqüente tende a iniciar-se com uma pausa e não com a emissão de algumas respostas (como pode ser visualizado nas marcas $b$ na Figura 4). O "running-through" 4 do responder do final de um intervalo de altas taxas para o início do intervalo posterior - sob uma contingência de FI de intervalos curtos de 10 s -, aparentemente contribuiu para que a distribuição do responder entre reforços sucessivos apresen-

4 Termo utilizado por Ferster e Skinner (1957) e Stoddard e cols. (1988) para se referir ao responder em taxa alta, que ocorre perto da liberação do reforço e que continua a ocorrer mesmo após a liberação do reforço. O termo em inglês foi mantido por não se encontrar uma tradução adequada para o português. tasse poucos padrões de scallop ou break-and-run e vários padrões que indicam um responder negativamente acelerado no início do intervalo (registro cumulativo de S23 e S24).

Em resumo, de modo geral, foi notada uma maior persistência no padrão comportamental quando a contingência mudou de FR para FI no desempenho dos participantes cujos pontos eram trocados por fotocópia ou dinheiro (Condições 1 e 2, respectivamente - Figura 2) do que para aqueles participantes cujos pontos não foram trocados por nada (Condição 3 - Figura 3). Os participantes da Condição 3 - Pontos que diminuíram a frequiência de respostas durante o FI tenderam a ganhar menos pontos do que os participantes que mantiveram o padrão de responder em taxa alta e constante (ver, por exemplo, o número total de respostas e total de pontos obtidos nos parênteses acima de cada registro cumulativo na Figura 3).

\section{Desempenho sob FI 10s dos participantes submetidos a uma história experimental de DRL}

A Figura 5 exibe a frequiência acumulada de pressão ao botão do participante S16 da Condição 2 - Dinheiro na terceira sessão de exposição ao programa de reforço em DRL 20 s e nas três sessões de reforço em FI 10s. O desempenho desse participante é representativo do desempenho de todos os participantes das três condições de reforço. Verifica-se que, na terceira exposição ao programa de reforço em DRL 20s (primeira coluna à esquerda da Figura 5), o padrão de resposta foi de taxa baixa sob o DRL 20s. A taxa de resposta aumentou ligeiramente durante as sessões de exposição ao programa de reforço em FI 10s.

Esse resultado sugere que o comportamento da maioria dos participantes com história de DRL ficou sob controle do parâmetro temporal do FI. Não foi possível analisar os dados os IRTs para os participantes das Condições 1 e 2 , porque eles foram submetidos às sessões experimentais com o software ProgRef v2, que não faz esse registro. A média dos IRTs dos participantes da Condição 3 - Pontos, durante a terceira exposição ao DRL 20s, foi de 26,1s para o participante S26; 24,4 s para o S27 e 26,4 s para o participante S28. A média dos IRTs diminuiu na terceira sessão de FI 10s, indicando que o padrão de responder desses participantes foi sensível à mudança do parâmetro temporal da nova contingência. A média dos IRTs na terceira sessão de exposição ao FI 10s foi de 11,2 s para o participante S26; 11,1s para o S27 e 12,1 s para o participante $S 28$. Inspeção visual dos registros cumulativos sugere que uma diminuição na média dos IRTs, semelhante às dos participantes da Condição 3 - Pontos, ocorreu para os participantes das Condições 1 e 2 .

Embora um efeito da história de reforço seja observado quando se compara a freqüência final de respostas na terceira sessão de exposição ao FI dos participantes dos Grupos FR e DRL (a freqüência final de respostas sob FI 10s dos participantes com história de responder sob FR - exceto o participante S25 - era maior do que a freqüência final sob FI 10s dos participantes com história de responder sob DRL), a contingência de FI 10s corrente parecia também exercer controle sobre o comportamento de todos os participantes do Grupo DRL (independentemente da condição de reforço) e de alguns dos participantes da Condição 3 - Pontos do Grupo FR. 


\section{Condição 2 - Dinheiro}

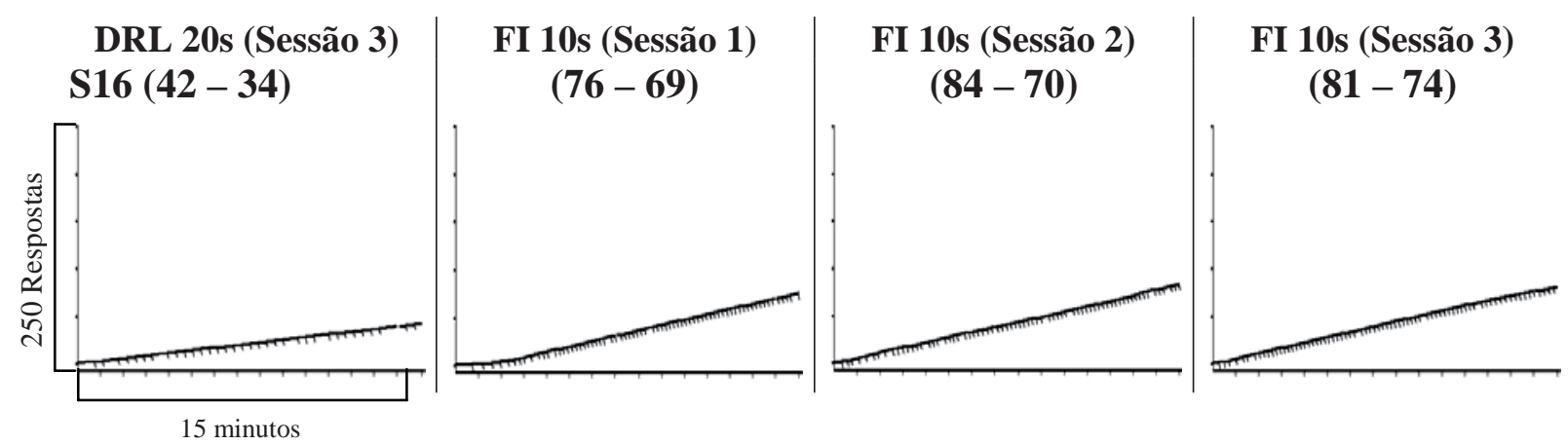

Figura 5. Freqüência acumulada de pressão ao botão do participante S16 (Condição 2-Dinheiro), na terceira sessão de exposição a um programa de reforço em DRL 20s (primeira coluna da esquerda) e nas três sessões de exposição a um programa de reforço em FI 10s. As marcas diagonais nos registros indicam a ocorrência de reforço. Todas as sessões tiveram duração de 15 minutos. Os números entre parênteses, acima de cada registro cumulativo, referem-se ao total de respostas e ao número de pontos obtidos naquela sessão.

Em linhas gerais, o tipo de reforçador empregado exerceu um efeito mais acentuado sobre o comportamento dos participantes com história de responder em FR do que sobre o comportamento dos participantes com história de responder em DRL quando submetidos a uma contingência de FI.

\section{Discussão}

\section{O responder sob FI dos participantes com história de FR e a influência do tipo de reforçador utilizado}

Os participantes com história de responder em FR apresentaram um padrão de responder em taxa alta e constante durante a terceira sessão do FR 40. A frequiência de respostas no FR foi independente do tipo de reforçador que os participantes recebiam (apenas o participante S3 da Condição 1 - Fotocópia exibiu um padrão de responder em taxa abaixo dos demais participantes, mas, mesmo assim, um desempenho de taxa alta). Esses resultados parecem em desacordo com aqueles relatados por Weiner (1972) em que taxa de resposta mais alta foi obtida quando os participantes, sob FR, trabalhavam por pontos trocáveis por dinheiro do que quando ganhavam o dinheiro por sessão, independente do número de pontos obtidos, ou quando trabalhavam por pontos apenas. Entretanto, Weiner manipulou o modo de pagamento intraparticipantes, cada participante era submetido a oito sessões de FR com cada uma das condições de pagamento. No presente estudo, o tipo de reforço foi manipulado interparticipantes. Talvez, em procedimentos no qual o reforço seja manipulado intraparticipantes, a história de responder, que produz uma determinada conseqüência, pode influenciar o comportamento subseqüente, sob a mesma contingência de reforço, quando a consequiência reforçadora é alterada.

Com relação ao efeito da história de responder em FR sobre o responder subseqüiente em FI, os participantes da Condição 1 - Fotocópia e da Condição 2 - Dinheiro exibiram um padrão de persistência comportamental semelhante àquele relatado por Weiner (1969; 1970). Embora os participantes com história de responder em FR que ganhavam pontos trocáveis por fotocópias (Condição 1) ou pontos trocáveis por dinheiro (Condição 2) tenham continuado a emitir taxa alta de resposta, mesmo quando as contingências mudaram para FI, a persistência no padrão comportamental não implicou a obtenção de menos reforçadores.

No caso do participante $\mathrm{S} 3$, a mudança na contingência implicou aumento no número de pontos obtidos (Cf. Figura 2). É possível levantar como hipótese que esse aumento seja devido à história de responder em FR e ao aumento na taxa de reforços quando a contingência mudou para FI 10s. O participante $\mathrm{S} 3$ emitiu 1.374, 1.202 e 1.538 respostas nas três primeiras sessões de FR, marcando 34, 30 e 38 pontos naquelas sessões (dados não exibidos nos resultados; mas consultar o Apêndice), ou seja, o participante teve uma história em que o aumento ou diminuição na freqüência total de resposta esteve correlacionada com um aumento ou diminuição no número total de pontos obtidos. Com a mudança da contingência de FR para FI, a taxa de reforços aumentou para esse participante, podendo ter contribuído para um aumento na taxa de resposta.

Quatro dos cinco participantes da Condição 3 - Pontos mudaram o padrão de responder de taxa alta e constante, na terceira sessão do FR, para um padrão de taxa relativamente menor, na terceira sessão do FI. Análises da distribuição das respostas entre reforços sucessivos, por inspeção visual dos registros cumulativos, indicaram que dois participantes (S22 e S23) apresentaram padrões de responder do tipo break-and-run em mais da metade dos intervalos entre reforços, fortalecendo a hipótese de que o responder desses participantes estava sob controle do parâmetro temporal do FI. O participante S24 exibiu um misto de responder negativamente acelerado e de taxa constante e o participante S25 exibiu principalmente um padrão de apenas uma resposta por intervalo. Os resultados dos participantes da Condição 3 - Pontos, com história experimental de responder sob FR, parecem de acordo com estudos conduzidos com não-humanos (Baron \& Leinenweber, 1995; Cole, 2001; Freeman \& Lattal, 1992; Urbain \& cols., 1978) que indicaram que a taxa de resposta tende a diminuir durante as exposições sucessivas dos sujeitos a um programa de reforço em FI, após uma história de responder em altas taxas.

Embora Weiner (1964) chame a atenção para a diferença na taxa final de respostas dos participantes com histórias de FR e DRL, os resultados obtidos com os participantes da Condição 3 - Pontos do presente experimento guardam alguma 
semelhança com aqueles observados no estudo de Weiner na medida em que dois dos três participantes do experimento de Weiner também diminuíram a taxa de resposta sob FI em relação à taxa que emitiram sob o FR precedente. Todavia, nenhum dos participantes do estudo de Weiner chegou a apresentar padrões de break-and-run como ocorreu com alguns participantes do presente experimento.

Em termos de procedimento, além do tipo de reforçador utilizado, outra diferença entre os experimentos de Weiner (1964, 1969, 1970) e o presente experimento foi a exigência de uma resposta de consumação para que os pontos fossem creditados no contador de pontos. De fato, os registros cumulativos de alguns participantes da Condição 3 - Pontos sob FI - após história de responder em FR - são semelhantes àqueles obtidos por Matthews e cols. (1977) e assemelham-se a alguns dos registros cumulativos observados no estudo de Buskist e cols. (1980), que também utilizaram uma resposta de consumação. Entretanto, os participantes cujos pontos eram trocáveis por dinheiro (Condição 2) ou fotocópias (Condição 1) exibiram um padrão de persistência comportamental, mesmo com a exigência da resposta de consumação. Portanto, a exigência de uma resposta de consumação talvez seja uma variável importante, mas parece não ser suficiente para produzir desempenho em taxa baixa sob FI, após uma história de reforço sob FR, em humanos. Como no presente experimento não havia uma condição em que a resposta de consumação estivesse ausente, dados experimentais que manipulem diretamente essa variável (presença vs. ausência de uma resposta de consumação) precisam ser produzidos para que se torne mais clara a importância dessa condição.

Também é preciso que a programação da resposta de consumação utilizada no presente estudo seja revista - como apontado nos resultados - para que dados mais claros do padrão de responder entre reforços possa emergir. O estudo de Matthews e cols. (1977) com humanos, e que utilizou uma resposta de consumação, não deixa claro se, uma vez que o reforço estava disponível, o botão de resposta era ou não desativado (ficava inoperante). Mas as luzes associadas ao botão de resposta eram desligadas e uma lâmpada associada ao botão de liberação do reforço era ligada. Esse arranjo pode ter sido suficiente para produzir uma interrupção na resposta operante tão logo a luz do botão de resposta fosse desligada, não gerando o "running-through" do responder no final de um intervalo sobre o início do intervalo subseqüente, observado no presente experimento. Um novo arranjo na programação da resposta de consumação talvez possa eliminar a grande quantidade de intervalos com responder negativamente acelerado observado no registro de alguns participantes.

Todavia, é preciso salientar que o "running-through" observado nos registros cumulativos - especialmente sob FI - de alguns participantes do presente experimento (S21, S24, S36) foi observado também por Ferster e Skinner (1957) no registro cumulativo de pombos respondendo sob FI. No caso dos experimentos de Ferster e Skinner, esses "runningthroughs" (cinco a 20 respostas ocorriam após a liberação do reforço) não chegavam a afetar substancialmente a distribuição das respostas dentro do intervalo entre reforços, porque o parâmetro do FI era de vários minutos. No presente experimento, os intervalos do FI eram de poucos segundos e, portanto, umas poucas respostas no início do intervalo eram suficientes para acentuar um padrão de responder negativamente acelerado.
Comparando-se o padrão de persistência comportamental dos participantes com história de FR, das três condições de reforço utilizadas, os resultados sugerem que pontos trocáveis por dinheiro e pontos trocáveis por fotocópias são reforçadores mais prováveis do que pontos apenas, em gerar um padrão de persistência comportamental quando as contingências mudam de FR para FI.

Um aspecto importante é que todos os participantes do Grupo FR - independentemente da condição de reforço - variaram a freqüência total de resposta emitida entre a primeira e a terceira sessão de exposição ao FR e, conseqüentemente, obtiveram um menor número de pontos quando a freqüência total de pressões ao botão foi menor. Esses dados não foram exibidos na seção de "Resultados", mas podem ser consultados nos Apêndices. Portanto, esses participantes fizeram contato direto com a correlação, existente nos programas de razão, entre número total de respostas emitidas e os pontos obtidos em uma sessão. Essa história, somada ao fato de que a persistência do responder em taxa alta sob FI não implicou a obtenção de menos pontos (em relação às sessões de FR precedentes) e mais o fato de que o tipo de reforçador dos participantes da Condição 1 - Fotocópias e da Condição 2 - Dinheiro poderia ser de maior "valor", parece ter contribuído para o padrão de persistência comportamental observada para os participantes das Condições 1 e 2 .

Uma palavra de advertência: o "valor" reforçador de pontos trocáveis por dinheiro ou fotocópias e de pontos apenas não foi avaliado diretamente no presente experimento. Uma exposição dos participantes a um programa de reforço em PR (Cf. Hodos, 1961) poderia ter oferecido uma avaliação mais direta do "valor" relativo dos três tipos de reforço empregados. Nas considerações anteriores há uma suposição, não testada, de que pontos trocáveis por dinheiro ou fotocópias podem ter valor reforçador maior do que pontos apenas.

Em outras palavras, os resultados do presente estudo sugerem que determinados tipos de reforçadores (pontos apenas) podem aumentar a probabilidade de mudança comportamental, mesmo que a mudança envolva algum risco de perda de reforçadores. Outros tipos de reforçadores (dinheiro e fotocópias para estudantes universitários) podem ser mais prováveis de gerar um padrão de persistência comportamental quando as contingências mudam, desde que essa persistência comportamental não implique a perda substancial de reforçadores. Esta última afirmação poderia parecer contraditória com os resultados de Weiner $(1965 ; 1969 ; 1970)$, nos quais um padrão de persistência foi observado mesmo quando os participantes mudavam de um programa de reforço em FR para um programa de FI-custo, no qual os participantes perdiam pontos pela emissão de respostas entre reforços sucessivos. No entanto, o custo da resposta era baixo em relação ao reforço (100 pontos por reforço e um ponto perdido por cada resposta emitida entre reforçadores) e, mais importante que isso, os participantes ganhavam por sessão e não pelos pontos.

Resultados de Weiner (1969), com humanos; de Cole (2001) e Wanchisen, Sutphin, Balogh e Tatham (1998), com ratos, demonstraram que, após uma história de responder em FR, os sujeitos reduziram a taxa de resposta quando foram submetidos a um programa de reforço em DRL sub- 
seqüentemente. Uma diferença crucial nessa mudança nas contingências de reforço é que um padrão de persistência comportamental nesse caso implicaria nenhum reforço por sessão. Isso não ocorre quando as contingências são alteradas de FR para FI.

Portanto, o efeito da história parece ser uma função das contingências específicas - tanto das observadas na história quanto das atuais (Cf. LeFrancois \& Metzger, 1993 e Weiner, 1983) - e do tipo e da maneira como os reforçadores são obtidos. Aparentemente, as contingências envolvidas quando as pessoas ganham pela participação na sessão, independente dos pontos obtidos (Weiner, 1964; 1965; 1969), são diferentes daquelas nas quais as pessoas não têm nenhuma conseqüência programada pela participação nas sessões experimentais além dos pontos obtidos na sessão (como na Condição 3 do presente experimento).

\section{O responder sob FI dos participantes com história de DRL e a influência do tipo de reforçador utilizado}

O tipo de reforçador parece não ter influenciado visivelmente a taxa de resposta sob FI dos participantes que tiveram história de responder sob DRL. Os resultados do presente experimento indicaram que, após uma história de responder sob um programa de reforço em DRL, todos os participantes (independentemente da condição de reforço) continuaram a exibir um padrão de responder em taxa baixa quando submetidos a um programa de reforço em FI 10s. Embora o padrão de responder em taxa baixa tenha persistido com a mudança nas contingências de reforço, a taxa de resposta aumentou quando a contingência mudou. Esse aumento na taxa de resposta durante o FI - após uma história de responder em DRL - foi observado também nos estudos de Weiner $(1964,1969)$ com humanos; Baron \& Leinenweber (1995), LeFrancois e Metzger (1993) e Urbain et al. (1978) com ratos; e Freeman e Lattal (1992) com pombos.

Esses resultados sugerem que, embora a história de reforço em DRL tenha produzido um responder em taxa baixa de, geralmente, uma resposta por intervalo, as contingências presentes durante a exposição ao FI exerceram também algum controle sobre o responder dos participantes. Uma evidência disso foi que, no presente experimento, a média dos IRTs dos participantes da Condição 3 - Pontos, que era superior a 20s durante a terceira exposição ao DRL 20s, diminuiu para aproximadamente $11,5 \mathrm{~s}$ durante a terceira exposição ao FI $10 \mathrm{~s}$.

\section{Considerações Finais}

Tomados em conjunto, os resultados do presente estudo parecem sugerir que: a) o comportamento dos participantes foi controlado tanto pela história de reforço quanto pelas contingências presentes; b) o tipo do reforçador empregado pode favorecer o responder em taxa alta e constante sob FI após exposição a uma contingência de FR e que, portanto, o tipo do reforçador pode ser uma variável importante para modular os efeitos da história experimental sobre o comportamento de seres humanos. Uma extensão desse último resultado com não-humanos requer ainda investigação empírica.

Além disso, os resultados do presente estudo assemelham-se àqueles obtidos em outros estudos com organismos humanos e não-humanos e sugerem que as diferenças entre o comportamento de humanos e não-humanos sob programas de reforço podem ser atribuídas tanto à história de condicionamento quanto a diferenças de procedimento entre os estudos com humanos e não-humanos.

\section{Referências}

Baron, A. \& Leinenweber, A. (1995). Effects of a variable-ratio conditioning history on sensitivity to fixed-interval contingencies in rats. Journal of the Experimental Analysis of Behavior, 63(1), 97-110.

Baum, W. M. (1999). Compreender o Behaviorismo: ciência, comportamento e cultura. (M. T. A. Silva, M.A. Matos, G.Y. Tomanari \& E.Z. Tourinho, Trad.). Porto Alegre: Artes Médicas. (Trabalho original publicado em 1994)

Buskist, W. F., Miller, H. L. \& Bennett, R. H. (1980). Fixed-interval performance in humans: sensitivity to temporal parameters when food is the reinforcer. Psychological Record, 30, 111-121.

Catania, A. C. (1998). Learning (4 ${ }^{\mathrm{a}}$ ed.). New Jersey: Prentice Hall.

Cole, M. R. (2001). The long-term effect of high- and low-rate responding histories on fixed-interval responding in rats. Journal of the Experimental Analysis of Behavior, 75(1), 43-54.

Costa, C. E. \& Banaco, R. A. (2002a). ProgRef v2: um software para o estudo de programas de reforço com humanos. Anais do XI Encontro Brasileiro de Psicoterapia e Medicina Comportamental. Londrina: ABPMC, p. 235.

Costa, C. E. \& Banaco, R. A. (2002b). ProgRef v3: Sistema computadorizado para coleta de dados sobre programas de reforço com humanos - recursos básicos. Revista Brasileira de Terapia Comportamental e Cognitiva, 4(2), 173-192.

Costa, C. E. \& Banaco, R. A. (2003). ProgRef v3: sistema computadorizado para a coleta de dados sobre programas de reforço com humanos - recursos adicionais. Revista Brasileira de Terapia Comportamental e Cognitiva, 5(2), 219-229.

Critchfield, T. S., Schlund, M. \& Ecott, C. (2000). A procedure for using bonus course credit to establish points as reinforcers for human subjects. Experimental Analysis of Human Behavior Bulletin, 18, 15-18.

Cumming, W. W. \& Schoenfeld, W.N. (1958). Behavior under extended exposure to a high-value fixed interval reinforcement schedule. Journal of the Experimental Analysis of Behavior, 1, 245-263.

Ferster, C. B. \& Skinner, B. F. (1957). Schedules of Reinforcement. New York: Appleton Century Crofts.

Freeman, T.J. \& Lattal, K.A. (1992). Stimulus control of behavioral history. Journal of the Experimental Analysis of Behavior, 57(1), 5-15.

Hodos, W. (1961). Progressive ratio as measure of reward strength. Science, 134, 943-944.

Lattal, K. A. (1991). Scheduling positive reinforcers. Em I. H. Iversen \& K. A. Lattal (Orgs.), Experimental Analysis of Behavior - Part 1 (pp. 87-134). Amsterdam: Elsevier.

LeFrancois, J. R. \& Metzger, B. (1993). Low-response-rate conditioning history and fixed-interval responding in rats. Journal of the Experimental Analysis of Behavior, 59(3), 543-549.

Lowe, C. F. (1979). Determinants of human operant behaviour. Em M. D. Zeiler \& P. Harzem (Orgs.), Reinforcement and the Organization of Behavior (pp. 159-192). New York: John, Wiley \& Sons.

Matthews, B. A.; Shimoff, E.; Catania, A. C. \& Sagvolden, T. (1977). Uninstrued human responding: sensitivity to ratio and interval contingencies. Journal of the Experimental Analysis of Behavior, 27(3), 453-467. 
Morse, W. H. \& Kelleher, R. T. (1970). Schedules as fundamental determinants of behavior. Em W.N. Schoenfeld (Orgs.), The Theory of Reinforcement Schedules (pp. 139-185). New York: Appleton-Century-Crofts.

Perone, M., Galizio, M. \& Baron, A. (1988). The relevance of animal-based principles in the laboratory study of human operant conditioning. Em G. Davey \& C. Cullen (Orgs.), Human Operant Conditioning and Behavior Modification (pp. 59-84). Chichester, England: John Wiley \& Sons Ltd.

Raia, C. P., Shillingford, S. W., Miller, H. L., Jr., \& Baier, P. S. (2000). Interaction of procedural factors in human performance on yoked schedules. Journal of the Experimental Analysis of Behavior, 74(3), 265-281.

Skinner, B. F. (1953). Science and Human Behavior. New York: The Free Press.

Skinner, B. F. (1966). Operant Behavior. Em W. K. Honig (Orgs.), Operant Behavior: Areas of Research and Application (pp. 12-32). New Jersey: Prentice-Hall.

Stoddard, L. T., Sidman, M. \& Brady, J. (1988). Fixed-interval and fixed-ratio reinforcement schedules with human subjects. Analysis of Verbal Behavior, 6, 33-44.

Tatham, T. A. \& Wanchisen, B. A. (1998). Behavioral history: a definition and some common findings from two areas of research. Behavior Analyst, 21(2), 241-251.

Urbain, C., Poling, A., Millan, J. \& Thompson, T. (1978). $d$-amphetamine and fixed-interval performance: effects of operant history. Journal of the Experimental Analysis of Behavior, 29(3), 385-392.

Wanchisen, B. A. (1990). Forgetting the lessons of history. Behavior Analyst, 13(1), 31-37.

Wanchisen, B. A., Sutphin, G. E., Balogh, S. A. \& Tatham, T. A. (1998). Lasting effects of a behavioral history of low-rate responding in rats. Learning and Motivation, 29, 220-235.

Wanchisen, B. A. \& Tatham, T. A. (1991). Behavioral history: a promising challenge in explaining and controlling human operant behavior. Behavior Analyst, 14(2), 139-144.
Wanchisen, B. A., Tatham, T. A. \& Mooney, S. E. (1989). Variable-ratio conditioning history produces high- and low-rate fixed-interval performance in rats. Journal of the Experimental Analysis of Behavior, 52(2), 167-179.

Weiner, H. (1964). Conditioning history and human fixed-interval performance. Journal of the Experimental Analysis of Behavior, 7(5), 383-385.

Weiner, H. (1965). Conditioning history and maladaptative human operant behavior. Psychological Reports, 17, 935-942.

Weiner, H. (1969). Controlling human fixed-interval performance. Journal of the Experimental Analysis of Behavior, 12(3), 349-373.

Weiner, H. (1970). Human behavioral persistence. Psychological Record, 20, 445-456.

Weiner, H. (1972). Human fixed-ratio responding as a function of the type of reinforcer (money $v$ s. points) and the presence or absence of a noncontingent monetary wage. Psychological Record, 21, 497-500.

Weiner, H. (1982). Histories of response omission and human operant behavior under a fixed-ratio schedule of reinforcement. Psychological Record, 32, 409-434.

Weiner, H. (1983). Some thoughts on discrepant human-animal performances under schedules of reinforcement. Psychological Record, 33, 521-532.

Zeiler, M. (1977). Schedules of reinforcement: the controlling variables. Em W. K. Honig \& J. E. R. Staddon (Orgs.), Handbook of Operant Behavior (pp. 201-232). New Jersey: Prentice Hall.

Recebido em 05.04.2006

Primeira decisão editorial em 06.09.2006

Versão final em 15.02.2007

Aceito em 20.03.2008 


\section{Apêndices}

Apêndice A. Frequiência total de pressão ao botão dos participantes em cada uma das sessões experimentais (exceto nas sessões de treino).

\begin{tabular}{|c|c|c|c|c|c|c|c|c|}
\hline \multirow[b]{3}{*}{ Reforço } & \multirow[b]{3}{*}{ Programa } & \multirow[b]{3}{*}{ Participante } & \multicolumn{3}{|c|}{ FR 40/DRL 20s } & \multicolumn{3}{|c|}{ FI 10s } \\
\hline & & & \multicolumn{3}{|c|}{ Sessões } & \multicolumn{3}{|c|}{ Sessões } \\
\hline & & & 1 & 2 & 3 & 1 & 2 & 3 \\
\hline Fotocópia & & $\mathrm{S} 1$ & 3768 & 4082 & 4038 & 4279 & 4218 & 4406 \\
\hline Fotocópia & & S2 & 2186 & 3459 & 3656 & 3647 & 3487 & 3408 \\
\hline Fotocópia & & S3 & 1374 & 1202 & 1538 & 2196 & 2346 & 2484 \\
\hline dinheiro & & S11 & 3538 & 3499 & 3611 & 3644 & 3624 & 3685 \\
\hline dinheiro & & S12 & 3764 & 3866 & 3900 & 3734 & 3494 & 4005 \\
\hline dinheiro & (FR)-FI & S13 & 3602 & 3188 & 3631 & 3551 & 3580 & 3631 \\
\hline Pontos & & S21 & 2371 & 2795 & 2776 & 2474 & 2661 & 2552 \\
\hline Pontos & & S22 & 3240 & 3275 & 3374 & 2591 & 189 & 296 \\
\hline Pontos & & S23 & 3395 & 3717 & 4227 & 2209 & 668 & 1450 \\
\hline Pontos & & S24 & 3883 & 4128 & 4471 & 4701 & 3086 & 675 \\
\hline Pontos & & S25 & 4074 & 3642 & 3398 & 395 & 92 & 83 \\
\hline Fotocópia & & $\mathrm{S} 4$ & 45 & 40 & 42 & 76 & 82 & --- \\
\hline Fotocópia & & S5 & 51 & 38 & 30 & 82 & 66 & 62 \\
\hline Fotocópia & & S6 & 42 & 36 & 49 & 72 & 77 & 76 \\
\hline dinheiro & & S14 & 62 & 41 & 31 & 36 & 33 & 60 \\
\hline dinheiro & (DRL)-FI & S15 & 165 & 39 & 40 & 49 & 84 & 77 \\
\hline dinheiro & & S16 & 56 & 42 & 42 & 76 & 84 & 81 \\
\hline Pontos & & S26 & 48 & 45 & 37 & 80 & 76 & 81 \\
\hline Pontos & & S27 & 47 & 38 & 37 & 69 & 77 & 83 \\
\hline Pontos & & S28 & 30 & 37 & 35 & 38 & 76 & 76 \\
\hline
\end{tabular}

Apêndice B. Total de pontos obtidos pelos participantes em cada uma das sessões experimentais (exceto nas sessões de treino).

\begin{tabular}{|c|c|c|c|c|c|c|c|c|}
\hline \multirow[b]{3}{*}{ Reforço } & \multirow[b]{3}{*}{ Programa } & \multirow[b]{3}{*}{ Participante } & \multicolumn{3}{|c|}{ FR 40/DRL 20s } & \multicolumn{3}{|c|}{ FI 10s } \\
\hline & & & \multicolumn{3}{|c|}{ Sessões } & \multicolumn{3}{|c|}{ Sessões } \\
\hline & & & 1 & 2 & 3 & 1 & 2 & 3 \\
\hline Fotocópia & & S1 & 94 & 102 & 100 & 88 & 88 & 88 \\
\hline Fotocópia & & S2 & 54 & 86 & 91 & 88 & 88 & 88 \\
\hline Fotocópia & & S3 & 34 & 30 & 38 & 87 & 87 & 87 \\
\hline Dinheiro & & S11 & 88 & 87 & 90 & 88 & 88 & 88 \\
\hline Dinheiro & & $\mathrm{S} 12$ & 94 & 96 & 97 & 88 & 88 & 88 \\
\hline Dinheiro & (FR)- FI & S13 & 90 & 79 & 90 & 88 & 88 & 88 \\
\hline Pontos & & $\mathrm{S} 21$ & 57 & 69 & 69 & 88 & 88 & 88 \\
\hline Pontos & & S22 & 81 & 81 & 84 & 82 & 70 & 79 \\
\hline Pontos & & S23 & 84 & 92 & 102 & 86 & 88 & 86 \\
\hline Pontos & & S24 & 96 & 103 & 110 & 89 & 88 & 87 \\
\hline Pontos & & S25 & 101 & 91 & 84 & 73 & 70 & 63 \\
\hline Fotocópia & & S4 & 34 & 35 & 36 & 71 & 77 & --- \\
\hline Fotocópia & & S5 & 23 & 35 & 25 & 65 & 61 & 59 \\
\hline Fotocópia & & S6 & 29 & 33 & 28 & 67 & 62 & 65 \\
\hline Dinheiro & & S14 & 27 & 36 & 29 & 34 & 33 & 54 \\
\hline Dinheiro & (DRL)-FI & S15 & 27 & 24 & 27 & 38 & 67 & 66 \\
\hline Dinheiro & & S16 & 28 & 34 & 34 & 69 & 70 & 74 \\
\hline Pontos & & S26 & 29 & 31 & 29 & 70 & 68 & 72 \\
\hline Pontos & & S27 & 33 & 33 & 34 & 61 & 67 & 74 \\
\hline Pontos & & S28 & 24 & 34 & 31 & 36 & 66 & 66 \\
\hline
\end{tabular}

